\title{
THE
}

$11-15-2007$

\section{Relaxation Time, Diffusion, and Viscosity Analysis of Model Asphalt Systems Using Molecular Simulation}

Liqun Zhang

Michael L. Greenfield

University of Rhode Island, greenfield@uri.edu

Follow this and additional works at: https://digitalcommons.uri.edu/che_facpubs

Part of the Chemical Engineering Commons

Terms of Use

All rights reserved under copyright.

\section{Citation/Publisher Attribution}

Zhang, L. \& Greenfield, M. L. (2007). Relaxation Time, Diffusion, and Viscosity Analysis of Model Asphalt Systems Using Molecular Simulation. Journal of Chemical Physics, 127(19), 194502. doi: 10.1063/ 1.2799189

Available at: http://dx.doi.org/10.1063/1.2799189

This Article is brought to you for free and open access by the Chemical Engineering at DigitalCommons@URI. It has been accepted for inclusion in Chemical Engineering Faculty Publications by an authorized administrator of DigitalCommons@URI. For more information, please contact digitalcommons-group@uri.edu. 


\section{AIP $\begin{gathered}\text { mesoumalot } \\ \text { chemical Physics }\end{gathered}$}

Relaxation time, diffusion, and viscosity analysis of model asphalt systems using molecular simulation

Liqun Zhang and Michael L. Greenfield

Citation: J. Chem. Phys. 127, 194502 (2007); doi: 10.1063/1.2799189

View online: http://dx.doi.org/10.1063/1.2799189

View Table of Contents: http://jcp.aip.org/resource/1/JCPSA6/v127/i19

Published by the American Institute of Physics.

Additional information on J. Chem. Phys.

Journal Homepage: http://jcp.aip.org/

Journal Information: http://jcp.aip.org/about/about_the_journal

Top downloads: http://jcp.aip.org/features/most_downloaded

Information for Authors: http://jcp.aip.org/authors

\section{ADVERTISEMENT}




\title{
Relaxation time, diffusion, and viscosity analysis of model asphalt systems using molecular simulation
}

\author{
Liqun Zhang ${ }^{\text {a) }}$ and Michael L. Greenfield ${ }^{\text {b) }}$ \\ Department of Chemical Engineering, University of Rhode Island, Kingston, Rhode Island 02881, USA
}

(Received 6 June 2007; accepted 21 September 2007; published online 15 November 2007)

\begin{abstract}
Molecular dynamics simulation was used to calculate rotational relaxation time, diffusion coefficient, and zero-shear viscosity for a pure aromatic compound (naphthalene) and for aromatic and aliphatic components in model asphalt systems over a temperature range of 298-443 K. The model asphalt systems were chosen previously to represent real asphalt. Green-Kubo and Einstein methods were used to estimate viscosity at high temperature $(443.15 \mathrm{~K})$. Rotational relaxation times were calculated by nonlinear regression of orientation correlation functions to a modified Kohlrausch-Williams-Watts function. The Vogel-Fulcher-Tammann equation was used to analyze the temperature dependences of relaxation time, viscosity, and diffusion coefficient. The temperature dependences of viscosity and relaxation time were related using the Debye-Stokes-Einstein equation, enabling viscosity at low temperatures of two model asphalt systems to be estimated from high temperature $(443.15 \mathrm{~K})$ viscosity and temperature-dependent relaxation time results. Semiquantitative accuracy of such an equivalent temperature dependence was found for naphthalene. Diffusion coefficient showed a much smaller temperature dependence for all components in the model asphalt systems. Dimethylnaphthalene diffused the fastest while asphaltene molecules diffused the slowest. Neat naphthalene diffused faster than any component in model asphalts. () 2007 American Institute of Physics. [DOI: 10.1063/1.2799189]
\end{abstract}

\section{INTRODUCTION}

Asphalts are naturally occurring mixtures composed of more than $10^{5}$ kinds of organic compounds. ${ }^{1}$ Based on solubility in different solvents, an asphalt can be classified into three parts: asphaltene, resin, and saturate. ${ }^{2}$ In the Corbett method, resin is further subdivided into naphthene aromatic and polar aromatic. Asphalt is widely used for road pavement and roof patching.

The Strategic Highway Research Program ${ }^{3,4}$ (SHRP) led to so-called "performance-graded" asphalts, which are rated for roadway applications based on the magnitude and temperature dependence of complex modulus and viscosity. ${ }^{2}$ Despite this common basis for initial mechanical performance, recent research has found some indications that comparably graded asphalts that differ in chemistry lead to different performance in roads. For example, asphalts of the same high temperature grading are reported to have performed differently in roadway studies. ${ }^{5}$ Asphalts of comparable complex modulus at the same upper temperature but rated for different lower temperatures exhibited different strains after repeated stress loadings (so-called "rutting") due to the resulting different temperature dependences of the loss compliance $J^{\prime \prime}{ }^{6}$

Current asphalt characterization methods are not yet capable of quantifying chemical effects on asphalt performance. This work is part of a larger project that uses model asphalts ${ }^{7,8}$ to investigate chemical effects; the specific intent here is to estimate the temperature-dependent change in zero-

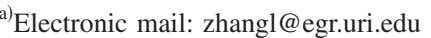

${ }^{b)}$ Electronic mail: greenfield@egr.uri.edu
}

shear asphalt viscosity, based on molecular-level changes in the dynamics of molecules within the model asphalt. Orientation relaxation rates are calculated using molecular dynamics simulations and are interpreted using a Debye-StokesEinstein approach.

Many previous studies have applied molecule-based interpretations to asphalt viscosity. Herrin and Jones ${ }^{9}$ related temperature and shear rate dependences using absolute rate theory. ${ }^{10}$ Khong et al. ${ }^{11}$ determined that asphalts of different sources and gradings each were best described by different parameters for the Williams-Landel-Ferry (WLF) equation. ${ }^{12}$ Viscosity extrapolations to lower temperatures showed poor correlation with other asphalt measures; this was attributed to elastic response. Christensen et al. ${ }^{13}$ attributed differences in viscosity of different SHRP asphalts to differences in free volume and intermolecular forces, with temperature dependence based on the WLF equation. Marasteanu and Anderson ${ }^{14}$ extended a model for asphalt complex modulus, comparing various temperature dependences for the shift factor in time-temperature superposition. They found that a WLF dependence was most appropriate between $T_{g}$ and $\left(T_{g}+100{ }^{\circ} \mathrm{C}\right)$. Below and above this range they recommended a WLF equation with a different reference temperature and an Arrhenius dependence, respectively. Zhai and Salomon ${ }^{15}$ used the Vogel-Fulcher-Tammann ${ }^{16-18}$ (VFT) and WLF equations to interpret asphalt viscositytemperature dependences measured experimentally. The asphalt binders they studied exhibited a non-Arrhenius dependence, behaving instead like a fragile liquid. ${ }^{19}$ Based on these findings, a non-Arrhenius dependence is expected for asphalt viscosity. 
Molecular dynamics is based on analysis of atom dynamics and interactions. It can predict the structure and thermodynamic properties of relatively complex fluids. ${ }^{20}$ Many researchers have used molecular dynamics (MD) simulation to study the viscosity temperature dependence of hydrocarbon compounds and fluids. For example, Mondello and Grest $^{21}$ used equilibrium molecular dynamics to calculate viscosity of $n$-alkanes with both the atomic and molecular stress representations and using both Green-Kubo and Einstein approaches; they found comparable viscosity results in all cases. In a later publication, ${ }^{22}$ they analyzed dynamics of $n$-alkanes and found that for chain length $n \geq 60$, dynamics analysis results (self-diffusion constant, viscosity, and equilibrium structure statistics) agreed with Rouse model estimates.

Using molecular dynamics simulation to calculate viscosity directly can be very time consuming. For simple liquids such as $n$-decane and $n$-hexadecane, simulations of order 70-200 times the rotational relaxation time were required to achieve a desired viscosity accuracy using Green-Kubo integration methods. ${ }^{21,23,24}$ As molecule size increased, Cui et $a l^{23,25}$ found that both this prefactor and the rotational relaxation time increased.

To circumvent such long simulations, several groups have proposed indirect estimation methods based on single molecules. Mondello and Grest ${ }^{21}$ found that viscosity scaled more similarly to rotational relaxation time than to diffusion coefficient, for moderate length alkanes. Bedrov et al. ${ }^{26}$ calculated liquid shear viscosity and self-diffusion coefficient of HMX (octahydro-1,3,5,7-tetranitro-1,3,5,7-tetrazocine) from a high temperature $(800 \mathrm{~K})$ to near the melting point $(550 \mathrm{~K})$ using equilibrium MD. Their results could be described by the Arrhenius equation over the entire temperature domain studied, with very similar apparent activation energies for self-diffusion and shear viscosity. They suggested estimating viscosity at low temperature via the self-diffusion coefficient and the Arrhenius equation, since calculating the selfdiffusion coefficient needs less computer time. Gordon ${ }^{24,27,28}$ investigated using Stokes-Einstein relationships to estimate pure fluid viscosity from self-diffusivity. He found that viscosity $\eta$ scaled with the temperature/diffusion coefficient ratio $T / D$ raised to a power slightly greater than unity. ${ }^{28}$ In addition, the product $D \eta / T$ varied with overall density and was relatively insensitive to temperature.

The rates of single molecule reorientations are determined by analyzing time correlation functions of segmental dynamics and their temperature dependences. For example, Budzien et al. ${ }^{29}$ analyzed the relationship of segmental dynamics with temperature and composition in a blend of alkanes. Using a united atom model, they found semiquantitative agreement between simulation and nuclear magnetic resonance (NMR) dynamics results for a moderate-size $\left(\mathrm{C}_{6}\right)$ alkane and very good agreement for a larger alkane $\left(\mathrm{C}_{24}\right)$. Comparable agreement was found in pure compounds and mixtures, validating the idea of using a simulation approach to infer dynamics in more complicated mixtures.

Here we present simulation results for complex multicomponent models of asphalt, ${ }^{8}$ which include $n$-alkanes, small aromatics, and complex aromatic compounds. Equilib- rium molecular dynamics was used to calculate viscosity, relaxation time, and self-diffusion coefficients. The GreenKubo and Einstein methods were used to calculate viscosity directly at high temperatures. Reorientation autocorrelation functions of single components were analyzed to calculate rotational relaxation times, which were then used to estimate viscosity at low temperatures using a Debye-StokesEinstein approach. Our goal of this work is to understand the relationship between temperature-dependent viscosity and local dynamics of molecules, comparing the impacts of two different asphaltene molecule structures. These results can ultimately provide insights on asphalt design and modification strategies.

\section{SIMULATION DETAILS}

\section{A. Ternary asphalt systems}

In earlier work, ${ }^{7,30}$ we devised two three-component systems as simple computational models of asphalt. We chose two model asphaltene molecules from the literature ${ }^{31,32}$ to represent an asphaltene component, 1,7-dimethylnaphthalene to represent resin (naphthene aromatic), and $n-\mathrm{C}_{22}$ to represent a saturate component. In follow-up work, ${ }^{33}$ we have considered a more sophisticated system that includes both types of resins. Overall composition was chosen to resemble the total $\mathrm{C} / \mathrm{H}$ ratio reported for a real asphalt. ${ }^{34,35}$ In the asphaltene1-based system, we used 5 of a model asphaltene molecule suggested by Artok et al., ${ }^{31} 27$ 1,7-dimethylnaphthalene, and $41 n$ - $\mathrm{C}_{22}$ molecules; in the asphaltene2based system, we used 5 of a model asphaltene molecule suggested by Groenzin and Mullins, ${ }^{32} 35$ 1,7-dimethylnaphthalene, and $45 n-\mathrm{C}_{22}$ molecules.

For the simulations, we chose the OPLS-aa forcefield, ${ }^{36,37}$ which is based on all-atom interactions, and used LAMMPS (version 2001) ) $^{38,39}$ to do parallel MD simulations. In order to analyze the temperature dependence of the model asphalt systems, four different temperatures were chosen: $298.15,358.15,400$, and $443.15 \mathrm{~K}$. We chose an allatom force field because united atoms have been shown ${ }^{40}$ to lead to incorrect molecular packing; such results were also of interest.

Atom configurations were initialized as in our prior work. ${ }^{7,8}$ Molecules were placed on a lattice, and isothermalisobaric Monte Carlo (TOWHEE program) (11,42 $^{\text {was used to }}$ eliminate the highest energy overlaps. Next we applied molecular dynamics at constant volume and temperature (NVT) using the velocity rescaling method for $50-100$ ps. This can dissipate initially high energies and helps to keep the system stable. Then we continued in the isothermal-isobaric (NPT) ensemble using the Nosé-Hoover thermostat and barostat ${ }^{20}$ for at least $2 \mathrm{~ns}$ to shrink system volume. After reaching the sampling state, in which systems showed consistent and steady instantaneous volume fluctuations around the average volume, we continued with NVT simulation (Nosé-Hoover) at the average volume using a $1.0 \mathrm{fs}$ time step. We simulated in the sampling state for 5-13 ns, depending on temperature (the lower the temperature, the longer the simulation time), collecting atom position data every 1 ps. 
TABLE I. $P_{3}$ relaxation time $\tau_{c}$ for $n-\mathrm{C}_{22}$ and dimethylnaphthalene molecules.

\begin{tabular}{cccc}
\hline \hline System & $\begin{array}{c}\text { Temperature } \\
(\mathrm{K})\end{array}$ & $\begin{array}{c}n-\mathrm{C}_{22} \\
(\mathrm{~ns})\end{array}$ & $\begin{array}{c}\text { Dimethylnaphthalene } \\
(\mathrm{ns})\end{array}$ \\
\hline Asphalt1 & 443.15 & 0.048 & 0.0047 \\
& 400 & 0.21 & 0.0119 \\
& 358.15 & 147.2 & 0.215 \\
& 298.15 & 182.8 & 1.934 \\
Asphalt2 & 443.15 & 0.042 & 0.0025 \\
& 400 & 0.11 & 0.0046 \\
& 358.15 & 25.95 & 0.037 \\
& 298.15 & 240.6 & 1.050 \\
\hline \hline
\end{tabular}

We chose the time step sizes by comparing energy drifts in NVE simulations at different average temperatures. Our test results showed that a large energy drift occurred for a time step of $2.0 \mathrm{fs}$. Total energy drifted much less with a time step of 1.5 fs but still showed large energy fluctuations. Time steps of 1.0 and $0.5 \mathrm{fs}$ led to similar energy conservation. In the NPT system, we used a time step of 0.5 fs to ensure system stability. In production runs we chose a $1.0 \mathrm{fs}$ time step to access longer times. NVT simulation was chosen for sampling dynamics since a barostat has been shown to influence dynamics results. ${ }^{43}$ Flexible bond lengths and angles were chosen since constraints can affect chain dynamics. ${ }^{44}$

At higher temperatures, the simulation procedure leads to good sampling, since the total simulation time exceeds the asphaltene relaxation time at 443.15 and $400 \mathrm{~K}$ (values are shown in Fig. 10). In addition, the 2 ns at constant pressure exceeds the relaxation time of the dimethylnaphthalene and $n-\mathrm{C}_{22}$ (see Table I). The relaxation of each asphaltene molecule thus occurs in an equilibrated environment of smaller molecules. At lower temperatures, only dimethylnaphthalene relaxes completely over the time scale of the simulation. Reported relaxation times are extrapolations based on the extent of relaxation that did occur.

\section{B. Relaxation time analysis}

Molecular motions lead to changes in molecule orientations. The reorientation correlation time can be described by a family of time-correlation functions as ${ }^{45}$

$$
C^{l}(t)=\left\langle P_{l}(\mathbf{u}(t) \cdot \mathbf{u}(0))\right\rangle .
$$

Here $\mathbf{u}$ is a unit vector, $P_{l}(x)$ is a Legendre polynomial, and $l$ is the degree number. For $l=1, P_{1}(x)=x$; for $l=2, P_{2}(x)$ $=\frac{1}{2}\left(3 x^{2}-1\right)$; and for $l=3, P_{3}(x)=\frac{1}{2}\left(5 x^{3}-3 x\right)$. Each function decays from 1 at time zero [perfect correlation, $|\mathbf{u}(0) \cdot \mathbf{u}(0)|$ $=1]$ to zero at long times, when the correlation function reaches a random value $\left[\langle\mathbf{u}(t) \cdot \mathbf{u}(0)\rangle=\langle\cos \theta\rangle=0,\left\langle\cos ^{2} \theta\right\rangle\right.$ $\left.=1 / 3,\left\langle\cos ^{3} \theta\right\rangle=0\right]$. The time correlation function $P_{1}$ corresponds to the spectral band shapes measured in infrared absorption. The $P_{2}$ function is related to NMR and Raman scattering experiments. ${ }^{29,45,46}$ The $P_{3}$ function relates to polarized Raman spectra. ${ }^{47}$

We chose the unit vector for analyzing local dynamics based on molecule structure and possible local motions.

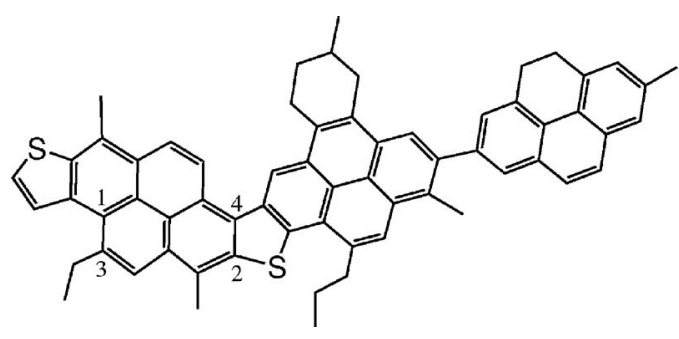

FIG. 1. Atoms used in asphaltene1 unit vector definitions.

$n-\mathrm{C}_{22}$ is like a short polymer chain. We used start and end carbon atoms to build the unit vector for calculating its relaxation time, because that vector shows the longest relaxation time for long chain molecules. ${ }^{48}$ Rotations of nonsymmetric molecules, such as asphaltenes, can be decomposed into independent motions about three principal axes, each with its own rate. ${ }^{46}$ For asphaltenes, these rates have been found to be similar. ${ }^{49}$ Tumbling motions that accompany shear flow require the fused aromatic rings of asphaltenes to change their geometry relative to the flow direction. Thus we choose the normal vector for monitoring rotational relaxations of aromatic compounds. Comparisons with relaxations of other vectors indicate that this choice is reasonable. ${ }^{50}$ The atom indices for asphaltene 1 and asphaltene 2 molecules are shown in Figs. 1 and 2. We used atoms $(1,2,3,4)$ to form two vectors $\mathbf{u}_{1-2}$ and $\mathbf{u}_{3-4}$. Then the normal vector was built as $\mathbf{u}=\left|\mathbf{u}_{1-2} \times \mathbf{u}_{3-4}\right|$. The equivalent atoms were used for dimethylnaphthalene.

Integrating the time correlation functions leads to different orders of characteristic times or rotational relaxation times $\tau_{l}$ (Ref. 45)

$$
\tau_{l}=\int_{0}^{\infty} C^{l}(t) d t
$$

The characteristic decay times for different values of $l$ are related by the Debye rule ${ }^{45}$

$$
\frac{\tau_{l}}{\tau_{l+1}}=\frac{l+2}{l}
$$

under the assumption that reorientation occurs as the result of a succession of small, uncorrelated steps. In order to test the Debye approximation for different orders of correlation functions, we can plot $-(l(l+1))^{-1} \log C^{l}(t)$ as a function of $t$. If the results satisfy the Debye approximation, those curves should overlap; otherwise, they deviate.

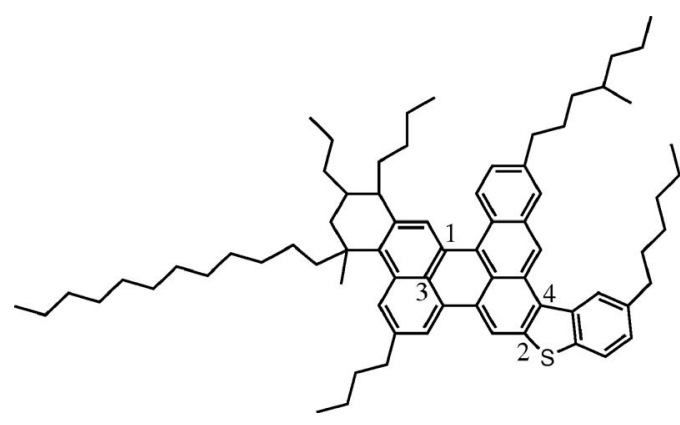

FIG. 2. Atoms used in asphaltene 2 unit vector definitions. 
After obtaining the correlation function, an alternative to using direct integration to calculate relaxation time is to regress the results using the modified Kohlrausch-WilliamsWatts (mKWW) function ${ }^{51,52}$

$P_{\mathrm{mKWW}}(t)=\alpha \exp \left(-t / \tau_{0}\right)+(1-\alpha) \exp \left(-\left(t / \tau_{\mathrm{KWW}}\right)^{\beta}\right)$.

The mKWW function has two characteristic times, $\tau_{0}$ and $\tau_{\mathrm{KWW}}$. The first typically describes the initial exponential decay of molecule orientation correlations, while the second describes the stretched exponential decay. $\alpha$ and $(1-\alpha)$ weigh the initial and stretched exponential decay contributions. The $\beta$ result indicates the width of stretched exponential decay. When pressure and composition are kept constant, those parameters typically have consistent relationships with temperature.

In order to observe the initial decay and stretched decay of correlation functions simultaneously, we analyzed the data following a method of Doxastakis et al. ${ }^{53}$ For the initial 10 ps decays, we collected atom position data every $0.1 \mathrm{ps}$ and averaged results from different restart points throughout the simulation. For correlations at longer times, we used atom position data collected every 1 ps. We merged these into a combined correlation function. Interpolation on a logarithmic scale then led to a data representation that is distributed more evenly in a log scale across the whole time domain than the original data, which ensures that equal attention is paid to initial and stretched decays. Deviations between the fit and simulation $P_{3}$ results were calculated on a linear scale.

Multiple ranges of time and $P_{3}(t)$ were chosen for fitting each interpolated data set. Simulation noise increased as $P_{3}(t)$ decreased, creating a trade-off such that eliminating more noise led to the data set terminating further above zero (i.e., at earlier times). Choosing different noise cutoffs enabled repeating a nonlinear regression of the KWW function process many times, using different initial guesses to obtain parameter values whose fitting results had very high correlation coefficients. The Levenberg-Marquardt algorithm in the program GRACE (version 5.1.12) ${ }^{54}$ was used. Ultimately we found that the same correlation function could be fit by different sets of parameters, achieving similar correlation coefficients and relaxation times, with exact parameter values being very sensitive to the chosen range of data. This process reduced any bias originating from different initial parameter values and enabled calculating average times and parameters. Arithmetic averaging was used for $\alpha$ and $\beta$, since the values obtained from different regressions spanned a narrow range. Relaxation times $\tau_{0}, \tau_{\mathrm{KWW}}$, and $\tau_{c}$ spanned wide ranges, so geometric averaging was used (i.e., arithmetic averaging of $\log \tau)$. Relaxation time was calculated by integration using the average parameters as

$$
\begin{aligned}
\tau_{c}=\int_{0}^{\infty} P_{\mathrm{mKWw}}(t) d t & =\alpha \tau_{0}+(1-\alpha) \tau_{\mathrm{KWw}} \frac{1}{\beta} \Gamma\left(\frac{1}{\beta}\right) \\
& \approx \int_{0}^{\infty} C^{l}(t) d t .
\end{aligned}
$$

Here $\Gamma$ is the gamma function. $\tau_{c}$ in this work corresponds to a mKWW function that describes the third order correlation function, $l=3$.

\section{Viscosity and diffusion coefficient}

In order to calculate viscosity $\eta$ of model asphalt systems, we used the Green-Kubo and Einstein methods, following approaches used earlier, ${ }^{21,24,55}$

$$
\begin{aligned}
& \eta=\frac{V}{10 k_{B} T} \int_{0}^{\infty}\left\langle\sum_{a, b} P_{a b}{ }^{\mathrm{st}}(0) P_{a b}{ }^{\mathrm{st}}(t)\right\rangle d t, \\
& \eta=\lim _{t \rightarrow \infty} \frac{d}{d t} \frac{V}{20 k_{B} T}\left\langle\sum_{a, b}\left(\Delta A_{a b}(t)\right)^{2}\right\rangle,
\end{aligned}
$$

where $\Delta A_{a b}(t)=\int_{0}^{t} P_{a b}{ }^{\text {st }}\left(t_{1}\right) d t_{1}$. Here $V$ is the system volume, $T$ is the temperature, and $k_{B}$ is the Boltzmann constant. The notation st indicates averaging equivalent instantaneous offdiagonal stress components and subtracting the pressure from the diagonal components, leading to a symmetrizedtraceless pressure tensor element $P_{a b}$ st. This stress-tensor correlation function $\left\langle P_{a b}{ }^{\text {st }}(0) P_{a b}{ }^{\text {st }}(t)\right\rangle$ was shown to improve convergence. ${ }^{21,55}$ The accuracy of these approaches was checked using the Green-Kubo and Einstein methods based on fluctuations of single off-diagonal elements. ${ }^{20} \mathrm{We}$ calculated the stress tensor using the molecular virial. ${ }^{56}$

The diffusion coefficient of each molecule type was calculated based on center of mass displacement ${ }^{57}$

$$
D=\frac{1}{2 d} \lim _{t \rightarrow \infty} \frac{\partial\left\langle x^{2}(t)+y^{2}(t)+z^{2}(t)\right\rangle}{\partial t} .
$$

Here $d$ is the dimensionality. We averaged over all three Cartesian components of the mean-squared displacement and over the center of mass displacements of all molecules of the same type to improve convergence.

\section{Temperature dependence of relaxation time, viscosity, and diffusion coefficient}

For temperatures above the glass transition temperature range, the molecular reorientation relaxation time and viscosity can be related based on the Debye-Stokes-Einstein relationship ${ }^{27,46}$

$$
\tau_{c}=K \frac{v_{p} \eta}{k_{B} T} .
$$

Here $v_{p}$ is the volume of the rotating molecule. $K$ is a prefactor that depends on the hydrodynamic boundary condition (stick or slip) and the molecule shape. For an asymmetric molecule like asphaltene, $K$ can be determined by experiment. For symmetric molecules, $K$ equals $1 .{ }^{46}$ Here we assume that $K$ is temperature independent and can be treated as a constant. We neglected the temperature dependence of single molecule volume at temperatures above the glass transition. Equation (9) thus suggests that relaxation time and the viscosity/temperature ratio scale similarly at different temperatures. According to our former analysis, ${ }^{7}$ the glass transition temperatures of model asphaltene1- and asphaltene2based systems should be below or around $298 \mathrm{~K}$. 
After calculating relaxation times at different temperatures, we can analyze its temperature dependence using the VFT equation $^{16-18}$

$$
\log \left(\tau_{c}\right)=\log \left(\tau_{\infty}\right)+\frac{B}{T-T_{0}} .
$$

Here $T_{0}$ is the glass transition temperature, $B$ is the apparent activation energy, and $\log \left(\tau_{\infty}\right)$ is the limiting value at high temperature. $T_{0}=0$ corresponds to the Arrhenius equation, which shows a linear $\log \tau_{c} \sim 1 / T$ relationship. If our relaxation time results are described well by the VFT equation, then we can use it to calculate their temperature dependence.

The temperature dependence of diffusion coefficients was also analyzed. The Rouse model ${ }^{48}$ relates viscosity and diffusion coefficient for chain molecules as

$$
\eta_{D}=\frac{\rho R T R_{g}{ }^{2}}{6 M D},
$$

where $\rho$ is the chain density, $M$ is molecular weight, $R_{g}{ }^{2}$ is the squared radius of gyration, and $\eta_{D}$ is the diffusionestimated viscosity. ${ }^{21}$ Connecting it with the Debye-StokesEinstein equation, we can see that as temperature changes, $\rho$ and $R_{g}{ }^{2}$ stay nearly constant, suggesting a consistent relationship among $\eta / T, \tau_{c}$, and $1 / D$. Such relationships have been employed previously by Mondello and Grest, ${ }^{21}$ Bedrov et $a l .{ }^{26}$ and Gordon, ${ }^{24,27,28}$ and they are studied here for the model asphalt systems. In the Rouse model, the viscosity is related to the longest relaxation time. Molecule rearrangements in response to stress occur until the longest relaxation time has elapsed. Thus in this work we assume that the temperature dependence of the longest relaxation time is related to the viscosity.

\section{SIMULATION TESTS}

The objective of this work is to relate overall relaxation, i.e., viscosity, to relaxation of single molecules: rotational correlation times and diffusion rates. Relaxation time can be calculated from different order Legendre polynomials of the correlation function. Equivalence among Legendre polynomials can be assessed using the Debye rule, Eq. (3). Results using the asphaltene 2 molecule in the model asphalt system at $358.15 \mathrm{~K}$ are shown in Fig. 3 as an example. Over times less than $100 \mathrm{ps}$, the first, second, and third order polynomials of the time correlation functions overlap very well and the deviations among them are very small (see inset). After $100 \mathrm{ps}$, the deviations increase. Up to $1 \mathrm{~ns}$, the deviations among them remain small. Beyond $1 \mathrm{~ns}$, the higher the order, the larger the deviation from $P_{1}$. We think the deviations at longer correlation times result in part from increased statistical uncertainty. When $P_{l}(t)$ becomes close to zero, the level of noise can overwhelm the small average signal. The Debye-rule test for small molecules is satisfied in a correspondingly shorter relaxation time.

The Debye rule results show an equivalency, within some accuracy, for different polynomial orders. Thus relaxation times of different order polynomials lead to the same time-temperature superposition behavior. This enables a choice about which order to use to calculate the relaxation

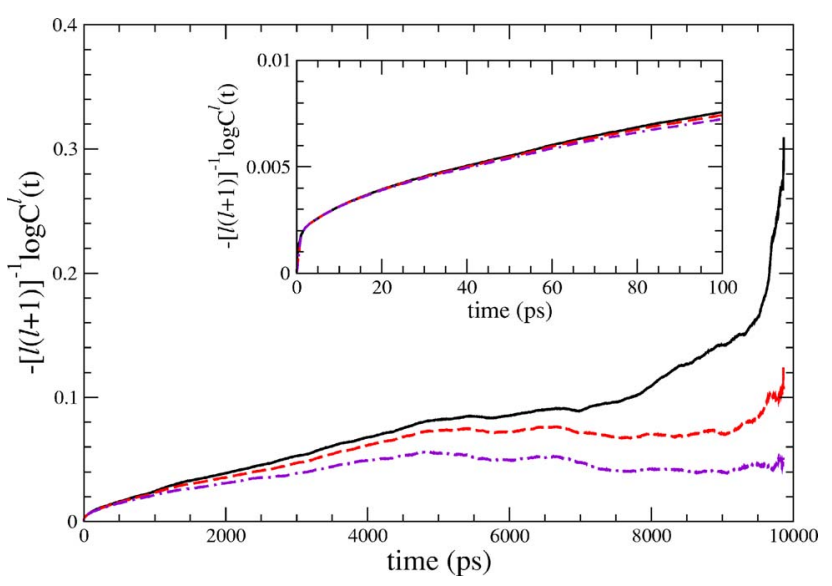

FIG. 3. (Color online) Debye rule test in the asphaltene2 system at $358.15 \mathrm{~K}$ for (solid line): the first order time correlation function $P_{1}$; (dashed line): $P_{2}$; (dot-dashed line): $P_{3}$.

time result in the shortest computation time. Based on these results, we have chosen to estimate the relaxation time $\tau_{1}$ for $\left\langle P_{1}(\mathbf{u}(t) \cdot \mathbf{u}(0))\right\rangle$ as $6 \tau_{3}$. Within the same amount of simulation time, $P_{3}$ can decay to zero the fastest and thus provides the most accurate correlation function.

\section{RELAXATION TIME, DIFFUSION, AND VISCOSITY FOR NAPHTHALENE}

Relaxation, viscosity, diffusion coefficient, and their temperature relationships were studied first for naphthalene. Naphthalene has aromatic rings similar to asphaltene molecules but a simpler structure, so we can calculate its relaxation time and viscosity results with more precision in a shorter computer time. Viscosity data available from the literature for naphthalene enable checking the accuracy of the simulation results, including the Green-Kubo method, the Einstein method, and the viscosity estimated based on the relaxation time and viscosity/temperature ratios (DebyeStokes-Einstein rule). These small molecule tests provide guidance for understanding asphaltene viscosity and relaxation time results.

The third order Legendre polynomial normal vector correlation function for naphthalene was calculated at four different temperatures: $298.15,358.15$, 400, and $458.15 \mathrm{~K}$. Results were regressed using the mKWW function and are shown in Fig. 4. At each temperature, the mKWW function describes the naphthalene unit vector correlation function decay very well. The naphthalene reorientation autocorrelations decay to zero in less than $50 \mathrm{ps}$ at all temperatures. As temperature increased from 298.15 to $458.15 \mathrm{~K}$, the decay rate increased.

The temperature dependence of the diffusion coefficient and mKWW function parameters are shown in Fig. 5. As temperature decreased from $458.15 \mathrm{~K}$ to $298.15 \mathrm{~K}, \beta$ decreased, while $\tau_{0}, \tau_{\mathrm{KWw}}, \tau_{c}$, and $1 / D$ increased. Relaxation times based on $P_{1}$ would be six times larger than those shown, using Eq. (3). The significant slower relaxations are described by $\tau_{0}$, while $\tau_{\mathrm{KWw}}$ encompasses a range of faster rates. Similar behaviors $\left(\tau_{0}>\tau_{\mathrm{KWW}}\right)$ have been reported for other systems. ${ }^{29}$ The decrease in $\beta$ indicates a broader range 


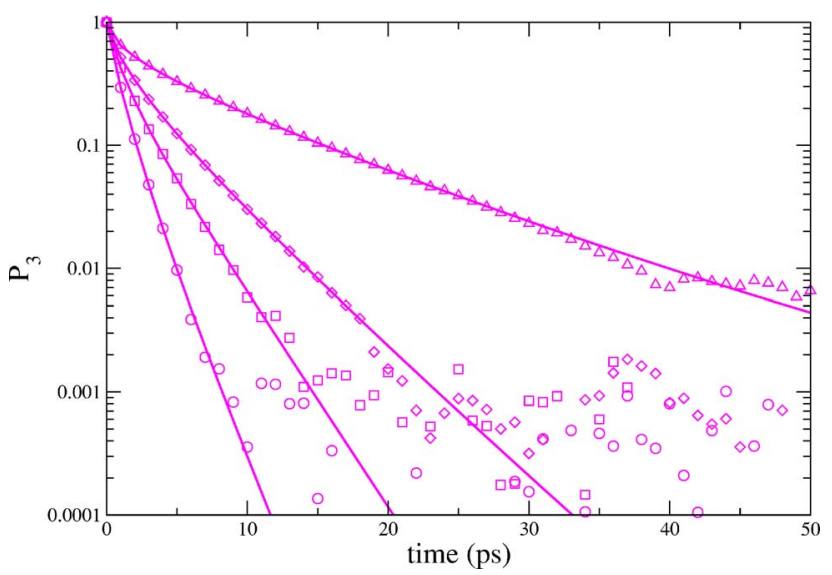

FIG. 4. (Color online) Naphthalene correlation function results at different temperatures, $\bigcirc: 458.15 \mathrm{~K} ; \square: 400 \mathrm{~K} ; \diamond: 358.15 \mathrm{~K}$; and $\triangle: 298.15 \mathrm{~K}$. Lines indicate $\mathrm{mKWW}$ fits. Points showing scatter at low $P_{3}$ values were not included in the mKWW fits due to their low signal-to-noise ratio.

of fast relaxation rates at lower temperatures. The overall naphthalene relaxation time $\tau_{c}$ and inverse diffusivity $1 / D$ are described well by an Arrhenius dependence (bold lines). Their slopes, which correspond to activation energy for relaxation and diffusion, respectively, show that the diffusion coefficient has a slightly larger temperature dependence than relaxation time.

Naphthalene viscosity was calculated using the GreenKubo and Einstein methods at temperatures of 358.15, 400, and $458.15 \mathrm{~K}$ as a function of increasing integration time, and results are shown in Fig. 6. Good agreement of viscosity results between the Green-Kubo and Einstein methods was observed at each temperature, with results from the GreenKubo method always slightly higher than those from the Einstein method. We read the viscosity estimate from the first plateau of the integrated correlation function beyond the overall molecule relaxation time, as shown in Fig. 6 using dashed lines. At $T=400 \mathrm{~K}$, naphthalene viscosity results are always higher than at $T=458.15 \mathrm{~K}$ but lower than the viscosity at $358.15 \mathrm{~K}$, at which we estimate a viscosity of around $0.90 \mathrm{cP}$ from the Green-Kubo method and $0.89 \mathrm{cP}$ from the Einstein method. We estimate viscosities of $0.57 \mathrm{cP}$

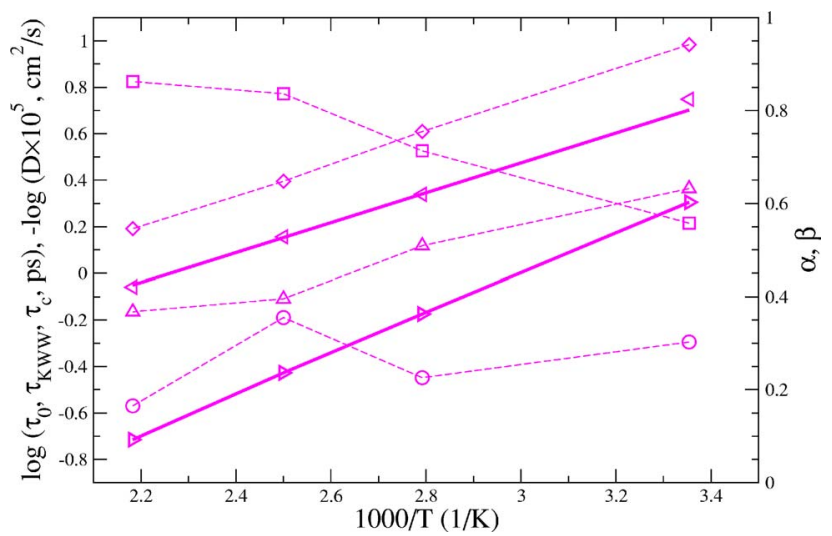

FIG. 5. (Color online) mKWW function parameters, overall relaxation time, and diffusion coefficient for naphthalene at different temperatures. Here $\bigcirc$ : $\alpha ; \square: \beta ; \diamond: \tau_{0} ; \triangle: \tau_{\mathrm{KWw}} ; \triangleleft: \tau_{c} ;$ and $\triangleright: 1 / D$. Solid lines indicate an Arrhenius dependence; dashed lines are guides for the eye.

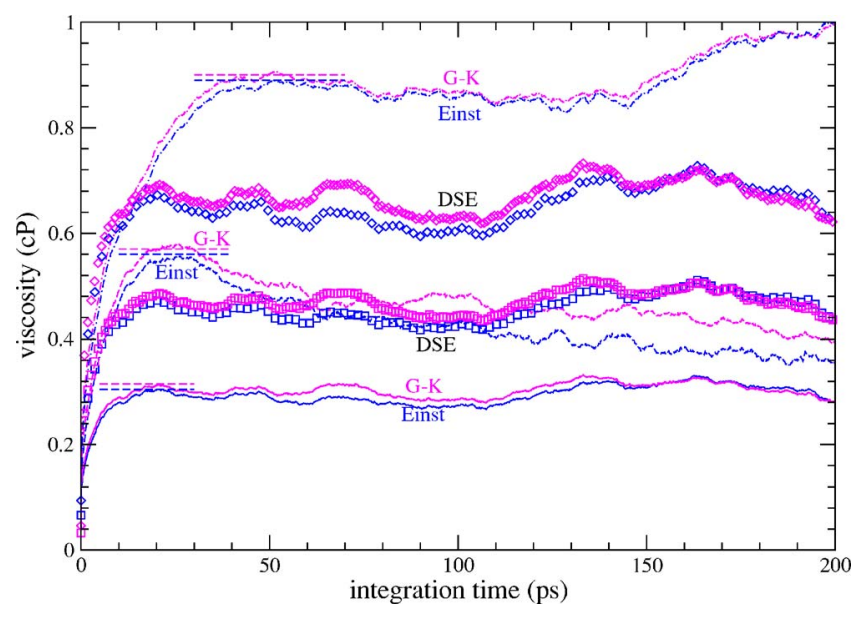

FIG. 6. (Color online) Cumulative naphthalene viscosity estimates using Eq. (6) at three different temperatures. Here, the solid line indicates simulation viscosity at $458.15 \mathrm{~K}$; the dashed line: simulation viscosity at $400 \mathrm{~K}$; the dot-dashed line: simulation viscosity at $358.15 \mathrm{~K}$. Symbols are estimated viscosity based on $458.15 \mathrm{~K}$ viscosity results at $400 \mathrm{~K}(\square)$ and $358.15 \mathrm{~K}$ $(\diamond)$. Dashed horizontal lines are the viscosity estimates.

and $0.56 \mathrm{cp}$ from the Green-Kubo and Einstein methods at $400 \mathrm{~K}$. At $458.15 \mathrm{~K}$ we estimate 0.32 and $0.31 \mathrm{cP}$ from the Green-Kubo and Einstein methods.

Temperature dependence of naphthalene viscosity is shown in Fig. 7. Experimental data ${ }^{58-61}$ have two selfconsistent Arrhenius-like temperature dependences. Differences between simulation results from the Green-Kubo and Einstein methods are shown as error bars. Those two methods always give similar results and these error bars do not account for uncertainty in the viscosity plateau value for either method. Interpolation of experimental data ${ }^{60}$ suggests a viscosity of $0.92 \mathrm{cP}$ at $358.15 \mathrm{~K}$. Our simulation estimates show remarkable agreement: lower by $1 \%-3 \%$. Analogous interpolation of other experimental data ${ }^{59}$ suggests a viscosity of $0.62 \mathrm{cP}$ at $400 \mathrm{~K}$; our simulation estimates are lower by $6 \%-10 \%$. Extrapolation suggests an experimental viscosity at $458.15 \mathrm{~K}$ of $0.36 \mathrm{cP}$; the simulation prediction is about $11 \%$ smaller. In total, the simulation predictions are very close to experimental data at two low temperatures while lower by $11 \%$ at $458.15 \mathrm{~K}$.

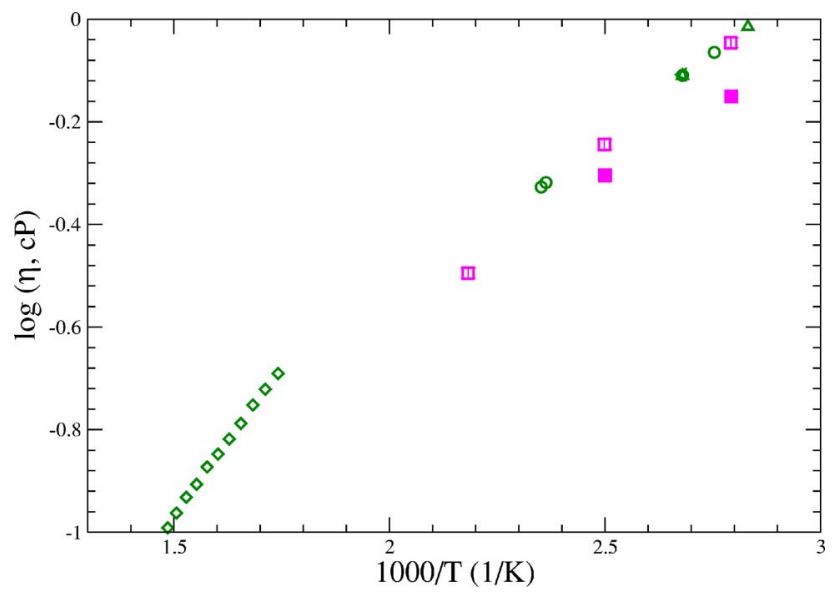

FIG. 7. (Color online) Naphthalene viscosity results ( $\square$ ) from simulation and reference data $\bigcirc$ (Ref. 58); $\diamond$ (Ref. 59); $\triangle$ (Ref. 60); and $\triangleleft$ (Ref. 61). Filled symbols ( $\square$ ) indicate viscosity estimated using $458.15 \mathrm{~K}$ results and relaxation time. 
Following Eq. (9), another comparison was made by using relaxation time, temperature, and molar volume ratios to estimate viscosity indirectly at 400 and $358.15 \mathrm{~K}$, with the $458.15 \mathrm{~K}$ viscosity as the base line. Indirect estimates and direct simulation results at $400 \mathrm{~K}$ have plateaus that are close to each other (Fig. 6); the indirect estimate is slightly lower over 10-60 ps of integration time but similar at longer times, particularly within the uncertainty of the direct simulation result. At $358.15 \mathrm{~K}$, the rise in indirect viscosity is comparable to the rise in direct viscosity estimate but reaches a lower plateau value. The indirect viscosity estimates are shown in Fig. 7 by filled symbols, while open symbols indicate direct Green-Kubo and Einstein viscosity predictions. The indirect 400 and $358.15 \mathrm{~K}$ viscosity estimates are lower than the experimental data, which are close to the direct results. The temperature dependence of the predictions based on the Debye-Stokes-Einstein equation can be approximately described by the Arrhenius equation and shows a temperature dependence slightly less steep than the experimental results. Differences between the indirect viscosity estimates and experimental data at lower temperatures can thus be attributed to the underestimate of viscosity at $458.15 \mathrm{~K}$. In total, the indirect estimates are reasonable considering the simulation uncertainties in calculating viscosity.

Viscosity results based on the Green-Kubo and Einstein methods have errors such as inherent simulation noise and ambiguity of the long time plateau value. For sufficiently long simulations, the stress fluctuations converge over time scales longer than the rotational relaxation time; then these errors are sufficiently small and a direct viscosity estimate is reasonable. At lower temperatures, the indirect estimate based on the Debye-Stokes-Einstein equation is more reasonable, because relaxation times obtained via fits to the mKWW function are less prone to errors than viscosity. These errors are sufficiently small in the longer $458.15 \mathrm{~K}$ simulations for naphthalene and a direct viscosity estimate is reasonable. In the shorter $400 \mathrm{~K}$ simulation, convergence of the direct calculation is supported by the eventual agreement between the direct and indirect viscosity estimates. Uncertainty in the direct estimate spans the range between the indirect estimate and the experimental viscosity. In the 358.15 $\mathrm{K}$ simulation, direct calculation leads to a viscosity estimate that is close to experiment, while the indirect estimate is somewhat lower.

Differences between the direct and indirect estimates result from simulation uncertainty and temperature dependence in the (uncalculated) prefactor. The naphthalene simulation results thus indicate that the present force field and equilibrium molecular dynamics simulation methods can lead to at least semiquantitative estimates of viscosity. Such estimates are valuable when viscosity is impossible to calculate directly, such as in model asphalts at low temperatures. With this framework in place, relaxation times and viscosities for model asphalts were pursued next.

\section{RELAXATION TIME IN MODEL ASPHALTS}

Rotation of individual molecules leads to relaxation of the unit vector correlation function. Describing the third Leg-

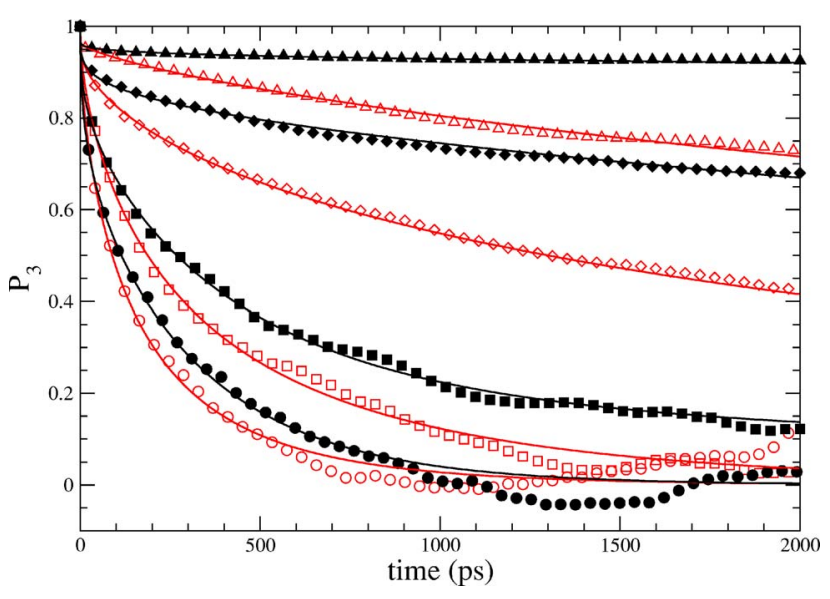

FIG. 8. (Color online) Changes in $\left\langle P_{3}(\mathbf{u}(t) \cdot \mathbf{u}(0))\right\rangle$ with correlation time for asphaltene 1 and asphaltene 2 molecules at different temperatures, $\bigcirc: 443.15$ $\mathrm{K} ; \square: 400 \mathrm{~K} ; \diamond: 358.15 \mathrm{~K}$; and $\triangle$ : $298.15 \mathrm{~K}$. Lines show fits using the mKWW function. Filled symbols represent asphaltene1; open symbols represent asphaltene2.

endre polynomial $P_{3}$ of each correlation function using the mKWW function leads to parameters $\alpha, \tau_{0}, \tau_{\mathrm{KWW}}$, and $\beta$. The rotational relaxation of each molecule type in model asphalt systems was analyzed for comparison with diffusion and viscosity results.

Figure 8 shows $P_{3}$ correlation results at four different temperatures for asphaltene 1 and asphaltene 2 molecules in the ternary model asphalt systems. Decays in the asphaltene normal vector correlation function are strongly temperature dependent: relaxation time decreased as temperature increased. The higher the temperature, the faster its local dynamics, so the faster its correlation function decays to zero. In all systems, the mKWW function describes $P_{3}$ correlation function relaxations well. At lower temperatures, the mKWW function provides a means for extrapolating the $P_{3}$ correlation function to zero at longer times than are accessible in direct simulations.

Multiple KWW parameter sets can describe each $P_{3}$ correlation function. Using the method described in Sec. II B, we obtained most probable parameters, relaxation times, and standard deviations, shown in Figs. 9 and 10. Standard de-

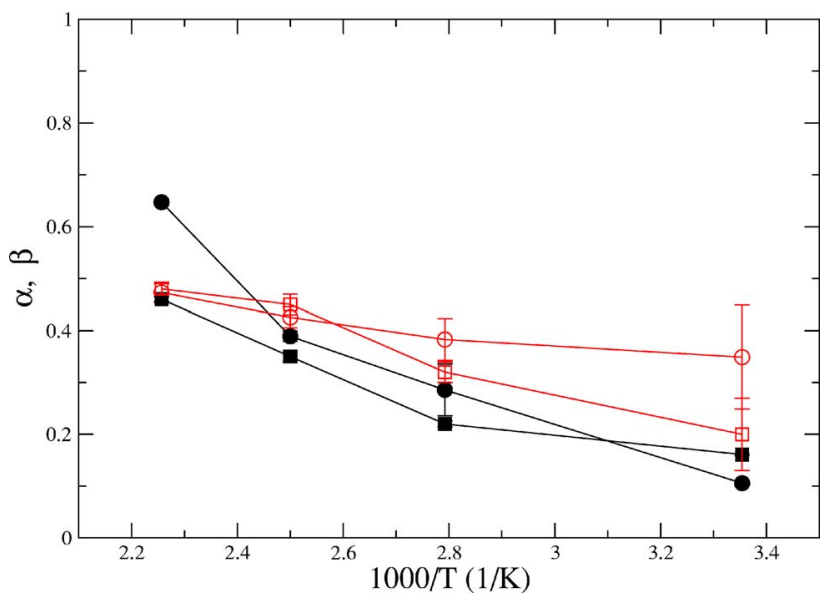

FIG. 9. (Color online) Change with temperature of $\alpha(\bigcirc)$ and $\beta$ ( $\square$ ) for asphaltene molecules. Filled symbols indicate asphaltene1 and open symbols asphaltene 2 molecules. 


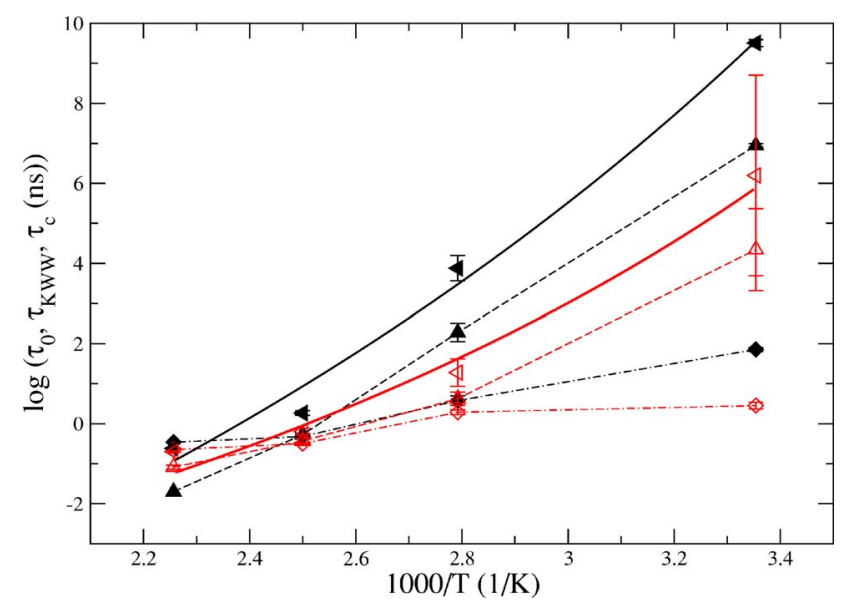

FIG. 10. (Color online) Change with temperature of $\tau_{0}(\diamond), \tau_{\mathrm{KWW}}(\triangle)$, and $\tau_{c}(\triangleleft)$ for asphaltene molecules. Filled symbols indicate asphaltene1 and open symbols asphaltene2 molecules. Dashed lines are guides for the eye while solid curves are fits to the VFT equation.

viations shown for relaxation times indicate relative errors and do not incorporate uncertainties for noise-dominated regions, such as beyond 500 ps for $P_{3}$ at $443.15 \mathrm{~K}$ in Fig. 8 . The lower the temperature, the higher the deviations in parameter values among the different possible regressions.

Figure 9 shows the temperature dependence of the average $\alpha$ and $\beta$ parameters for asphaltene molecules. As temperature decreases, $\alpha$ decreases for both molecules, indicating a larger contribution from the stretched exponential. $\alpha$ for asphaltene 1 shows a stronger temperature dependence than for asphaltene2. $\beta$ shows a similar temperature dependence in both asphalt systems, while the lower $\beta$ values in the asphaltene1 system suggest a wider range of relaxation rates.

Figure 10 shows the temperature dependence of the averaged time parameters for asphaltene molecules. Large increases in $\tau_{\mathrm{KWW}}$ and $\tau_{c}$ occur with decreasing temperature, while increases in $\tau_{0}$ are smaller. At temperatures lower than $400 \mathrm{~K}$, asphaltene 1 has larger $\tau_{0}, \tau_{\mathrm{KwW}}$, and $\tau_{c}$ values than asphaltene2, while at $443.15 \mathrm{~K}$ asphaltene 2 has a slightly larger $\tau_{\text {Kww }}$ parameter. VFT behavior has been observed experimentally for asphalts. ${ }^{15}$ Over a temperature range of 443.15-298.15 K, asphaltene1 and asphaltene2 relaxation time temperature dependences could be described by the VFT equation (solid lines). Activation energies of 14.7 and $27.7 \mathrm{~kJ} / \mathrm{mol}$ were found for asphaltene 1 and asphaltene2, respectively, with $T_{0}$ values of 146 and $168 \mathrm{~K}$. These values are approximately 100 degrees below the values we estimated from dilatometry simulations. ${ }^{7}$

Figure 10 indicates total relaxation times for asphaltenes at $443.15 \mathrm{~K}$ of $0.24 \mathrm{~ns}$ for the asphaltene 1 system and 0.20 $\mathrm{ns}$ for the asphaltene 2 system. These correspond to overall relaxation times $\tau_{1}$ of 1.44 and $1.2 \mathrm{~ns}$, using Eq. (3). Comparable relaxation times of 0.1 to $1.1 \mathrm{~ns}$ have been measured experimentally for asphaltene molecules in toluene solutions at room temperature. ${ }^{32,49}$ While the chemical environments and temperatures differ for these cases, the viscosities are similar $\left(0.59 \mathrm{cP}\right.$ in toluene solution, ${ }^{32,49} 1.10$ and $1.35 \mathrm{cP}$ for the asphaltene1 and asphaltene2 systems here; see Fig. 13).
Considering that relaxation time depends most strongly on viscosity [Eq. (9)], this agreement in relaxation time is satisfactory.

At all temperatures, asphaltene2 molecules decay faster than asphaltene 1 molecules over the first $1 \mathrm{~ns}$, as shown in Fig. 8. Considering that the asphaltene molecules are moving in similar chemical environments, ${ }^{8}$ the consistently faster decay rates for asphaltene 2 molecules indicate effects of molecular structure differences between the asphaltenes. Asphaltene1 has a larger fused ring structure with only short branches. Asphaltene2 molecules have longer aliphatic chains attached to moderate size aromatic rings. The larger aromatic ring size of asphaltene 1 makes its reorientation motion more slow, while the smaller fused ring structure of asphaltene 2 can relax faster. Fused aromatic ring correlations mostly decay via reorientation motions, while aliphatic chains can relax via conformational motions

Other molecules in model asphalts are smaller than asphaltenes and thus their orientations relax at faster rates. The relaxation time temperature dependence for dimethylnaphthalene and $n-\mathrm{C}_{22}$ were analyzed using a similar method as for asphaltene molecules, and overall results are shown in Table I. As temperature decreased from 443.15 to $298.15 \mathrm{~K}$, $\alpha$ and $\beta$ decreased for dimethylnaphthalene and $n-\mathrm{C}_{22}$ molecules in both asphalt systems. ${ }^{50}$ For $n$ - $\mathrm{C}_{22}$ molecules, $\tau_{0}$ and $\tau_{\mathrm{KWW}}$ increased by almost the same extent as the rotational correlation time $\tau_{c}$ : in the asphaltene1-based system by $10^{3.6}$ and in the asphaltene2-based system by $10^{3.8}$. For dimethylnaphthalene molecules, $\tau_{0}$ increased slightly more in the asphaltene1-based system than in the asphaltene2-based system; $\tau_{\mathrm{KWW}}$ increased to almost the same extent in both systems. $\tau_{c}$ increased accordingly, by $10^{2.6}$ in the asphaltene1and $10^{2.4}$ in the asphaltene2-based system. $n$ - $\mathrm{C}_{22}$ molecules have a stronger relaxation time temperature dependence in both systems than dimethylnaphthalene molecules, corresponding to a larger activation energy in the VFT equation. That may relate to the bigger size of $n-\mathrm{C}_{22}$ molecules. The different temperature dependences for different molecules emphasize the need to use the longest reorientation relaxation time of the slowest molecule for estimating the temperature dependence of viscosity.

Environment can influence the local dynamics of molecules and change their relaxation times. In model asphalt systems and in its pure state, the rotational correlation function of dimethylnaphthalene decays at different rates, as shown for 298.15 K in Fig. 11. Dimethylnaphthalene molecules in the pure state have the shortest relaxation time, $\tau_{c}$ $=48$ ps. The corresponding dimethylnaphthalene relaxation times increased in the model asphalt systems: $\tau_{c}=1050 \mathrm{ps}$ in the asphaltene2-based system and $\tau_{c}=1930 \mathrm{ps}$ in the asphaltene1-based system. Budzien et al. ${ }^{29}$ found similar changes in relaxation time for $\mathrm{C}_{6}$ and $\mathrm{C}_{24}$ alkanes. The reason is that for a molecule placed in a more viscous solvent, its dynamics are slowed down by bigger surrounding molecules. The higher the viscosity of the medium, the longer the relaxation time of dimethylnaphthalene, which is consistent with the Debye-Stokes-Einstein relationship. The overall rotational relaxation time $\tau_{1} \approx 6 \tau_{c}$ for dimethylnaphthalene in the model asphalts at temperatures above $298.15 \mathrm{~K}$ is 


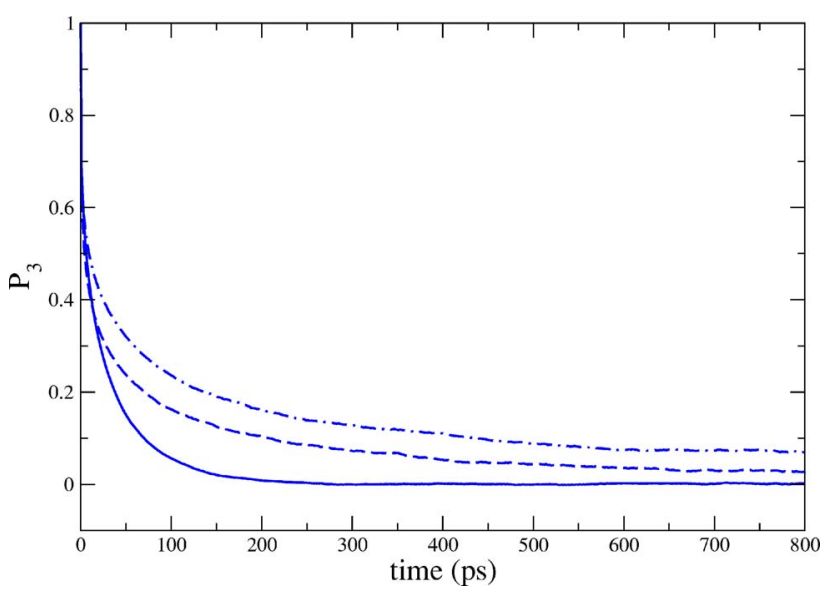

FIG. 11. (Color online) Rotational correlation function for dimethylnaphthalene at $T=298.15 \mathrm{~K}$ in (solid line) pure dimethylnaphthalene, (dashed line) asphaltene2-, and (dot-dashed line) asphaltene1-based systems.

much less than the simulation time at constant pressure $(2$ $\mathrm{ns}$ ), as is $\tau_{1}$ for $n-\mathrm{C}_{22}$ at 458.15 and $400 \mathrm{~K}$. The dimethylnaphthalene (and $n-\mathrm{C}_{22}$ to some extent) is thus able to equilibrate the environment surrounding asphaltene molecules before sampling of the orientation correlation function begins.

\section{DIFFUSION COEFFICIENTS IN MODEL ASPHALTS}

As a further probe of single-molecule dynamics, we analyzed the diffusion coefficients of each molecule type in the model asphalt systems. Figure 12 shows that as temperature decreased, the diffusion coefficient of each component decreased. Asphaltene1 and asphaltene 2 molecules diffused the slowest, due to their large size, while dimethylnaphthalene molecules have the smallest size and diffused the fastest. $n-\mathrm{C}_{22}$ molecules are chains and their diffusion rate is in between. Each component in the model asphalt systems diffused much slower than naphthalene. That relates to its smaller size and viscosity.

In similar chemical environments, asphaltene 1 and asphaltene 2 molecules diffuse at similar rates except at 298.15

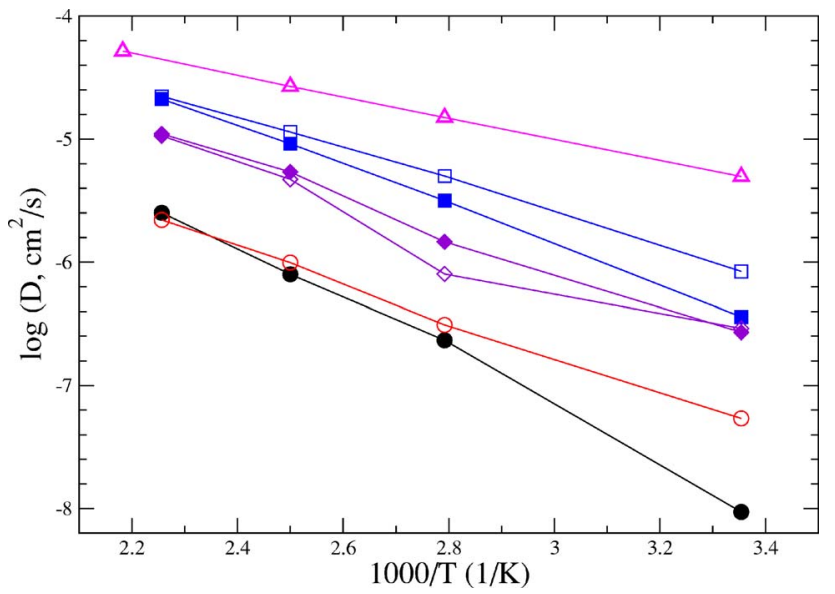

FIG. 12. (Color online) Diffusion coefficients of naphthalene and components in model asphalts. $\triangle$ : naphthalene; $\square$ : dimethylnaphthalene; $\diamond$ : $n-\mathrm{C}_{22}$; and $\bigcirc$ : asphaltene. Filled and open symbols indicate asphaltene1- and asphaltene2-based systems, respectively.
K. There, asphaltene1 diffused slower than asphaltene2, which may relate to poor statistics from simulation data at low temperatures. $n-\mathrm{C}_{22}$ molecules diffuse faster in the asphaltene1-based system than in the asphaltene2-based system at intermediate temperatures and the diffusion coefficient temperature dependences are very similar. That may relate to the medium and the molecular structure of $n-\mathrm{C}_{22}$. $n-\mathrm{C}_{22}$ molecules are long chains, compared to the two aromatic compounds. With fewer obstacles from aliphatic chains bonded to asphaltenes, they can diffuse in the asphaltene1 system more easily. The higher the temperature, the faster its diffusion. The diffusion coefficient was approximately $20 \%-40 \%$ of the value that can be estimated from experimental data for pure alkanes, ${ }^{62}$ where its activation energy is two thirds of that found here. In the asphaltene1based system, dimethylnaphthalene molecules diffused slower than in the asphaltene2-based system, which may relate to the molecular structure of asphaltene2. We calculated that the long aliphatic sidechains of asphaltene 2 lead to extra accessible volume. This extra space is enough for faster diffusion of dimethylnaphthalene but is not enough to affect diffusion of $n-C_{22}$. Accessible volume analysis ${ }^{63}$ of model asphalt systems will be the subject of a future paper. ${ }^{64}$

\section{VISCOSITY ESTIMATION OF MODEL ASPHALTS}

In order to use the Green-Kubo and Einstein methods to calculate viscosity from molecular dynamics simulation for a complex fluid like asphalt, it is useful to estimate the simulation time required. Literature data suggest a viscosity range of $3.7 \times 10^{7}-3.8 \times 10^{8} \mathrm{cP}$ at room temperature for SHRP core asphalts. ${ }^{65}$ Using the Debye-Stokes-Einstein equation, we can estimate that the relaxation time range within model asphalts reaches $0.06 \mathrm{~s}$ for asphaltene molecules.

This estimated relaxation time greatly exceeds available simulation time. Based on Cui et al.'s analysis, ${ }^{23}$ the time simulated should be much longer than a single relaxation time in order to attain accurate viscosity results from GreenKubo methods. Thus it is not possible to calculate asphalt viscosity directly at low temperatures. For example, long simulations (exceeding $12 \mathrm{~ns}$ ) for the asphaltene1-based system at $358.15 \mathrm{~K}$ did not converge to a well-defined viscosity value. This is consistent with its long relaxation time (7.5 $\times 10^{3} \mathrm{~ns}$ ); see Fig. 10. That is the motivation for using relaxation time and temperature ratios to estimate low temperature viscosity via the Debye-Stokes-Einstein relationship.

The shorter relaxation times at higher temperatures enable the viscosity to be calculated directly from simulation for asphaltene1- and asphaltene2-based systems at $443.15 \mathrm{~K}$. Running for more than 8 ns leads to estimates of the molecular virial-based viscosity [Eq. (6)] shown in Fig. 13. Asphaltene1- and asphaltene2-based systems have similar viscosities of 1.1 and $1.35 \mathrm{cP}$ at $443.15 \mathrm{~K}$. At $400 \mathrm{~K}$, the asphaltene1-based system has a viscosity $(2.2 \mathrm{cP})$ similar to that of the asphaltene2-based system $(2.1 \mathrm{cP})$, based on the approximately $\tau_{\text {tot }} / 6 \tau_{3}=0.72$ and 2.1 relaxation times that could be achieved for asphaltene 1 and asphaltene 2 using 


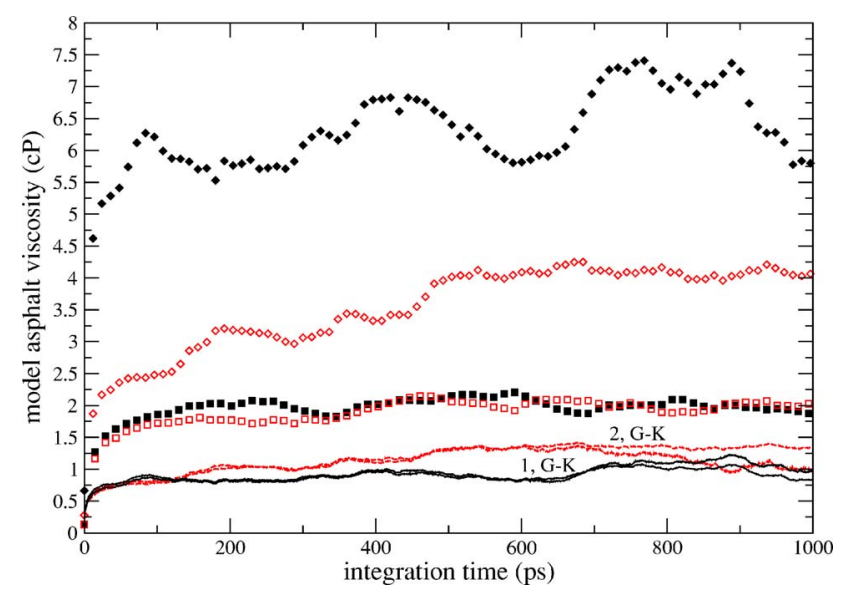

FIG. 13. (Color online) Viscosity results at 443.15 and $400 \mathrm{~K}$ for different asphalt systems from direct simulation and indirect estimation. Here the direct simulation result at $443.15 \mathrm{~K}$ for asphaltene1- (solid line) and for asphaltene2- (dashed line) based systems, $\square$ : direct simulation results at 400 $\mathrm{K}$, and $\diamond$ : indirect estimates at $400 \mathrm{~K}$ from $443.15 \mathrm{~K}$ results and relaxation times. Filled symbols are for asphaltene1 and open symbols for asphaltene2. At $443.15 \mathrm{~K}$, viscosity simulation results from both the Green-Kubo and Einstein methods are shown. At $400 \mathrm{~K}$, viscosity results from the GreenKubo method are shown.

available computational resources. At $400 \mathrm{~K}$, these relative times are too short to converge a meaningful viscosity estimate.

In order to determine if rotational relaxation time and/or diffusion coefficient could lead to accurate viscosity estimates, we first compared their temperature dependence. Figures 10 and 12 show that over the temperature range of 443.15-298.15 K, $\tau_{c}$ increased by $\tau_{298} / \tau_{443}=10^{10.1}$, while $D$ decreased by $10^{2.3}$ for asphaltene 1 molecules; $\tau_{c}$ increased by $10^{6.9}$, while $D$ decreased by $10^{2.3}$ for asphaltene 2 molecules. Diffusion coefficients showed a much smaller temperature dependence. Over 443-298.15 K, the diffusion coefficient of $n-\mathrm{C}_{22}$ in both asphaltene1- and asphaltene2-based systems decreased by $10^{1.5}$, while relaxation time increased by $10^{3.6}$ and $10^{3.8}$, respectively. The diffusion coefficient has a slightly smaller temperature dependence than the rotational relaxation time. For dimethylnaphthalene in both model asphalts over 443.15-298.15 K, the diffusion coefficient decreased by $10^{1.5}$, while relaxation time increased by $10^{2.6}$ and $10^{2.4}$, respectively. Diffusion coefficient again showed a smaller temperature dependence. Asphaltene rotational relaxation time shows the largest dependence and thus we consider it the most appropriate for estimating model asphalt viscosity.

The viscosity results calculated at 443.15 and $400 \mathrm{~K}$ using the Green-Kubo method can be compared to the Debye-Stokes-Einstein equation predictions as shown in Fig. 13, using the relaxation times $\tau_{c}$ for the asphaltene 1 and asphaltene 2 molecules shown in Fig. 10. Direct viscosity calculations from Green-Kubo and Einstein methods at 443.15 $\mathrm{K}$ from both systems are shown in Fig. 13, while estimates at $400 \mathrm{~K}$ came from the Green-Kubo method. Good agreement between those two methods was observed. Assuming temperature independence of the molecule shape terms (prefactor in the Debye-Stokes-Einstein equation) and neglecting changes in molecular volume leads to viscosity ratios be-

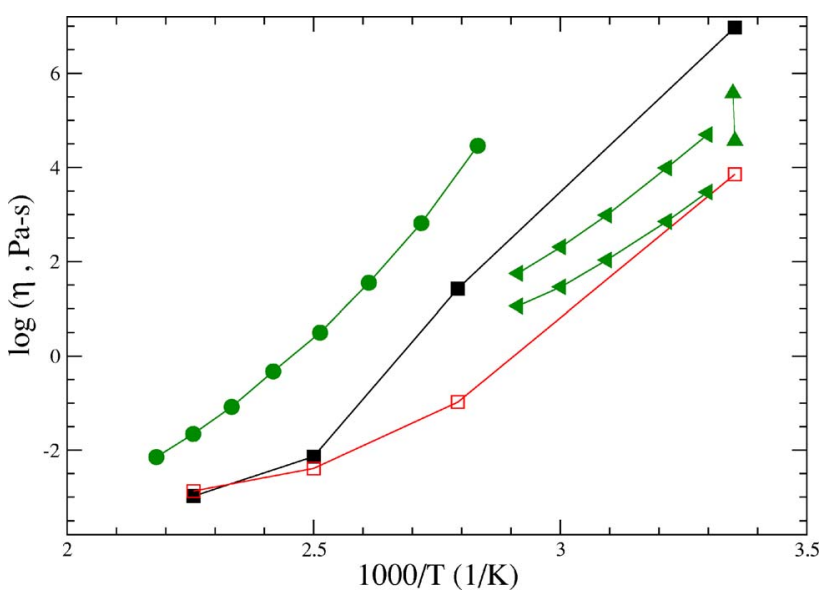

FIG. 14. (Color online) Viscosity comparison among indirect simulation predictions using Eq. (9) for $\square$ : asphaltene1 (filled) and asphaltene2 (open); -: experimental results on a PG 64-22 asphalt (Ref. 15); $\mathbf{\Delta}$ : experimental range for SHRP core asphalts (Ref. 65); and ४: range of experimental results reported for several penetration-graded asphalts (Ref. 11).

tween 443.15 and $400 \mathrm{~K}$ of 7.7 and 3.35 for the asphaltene1and asphaltene2-based systems. The resulting estimates of $400 \mathrm{~K}$ viscosity results are shown for both systems in Fig. 13. For the asphaltene1-based system, direct viscosity calculation (filled squares) only reaches one third of the viscosity estimated using the relaxation time-temperature prediction [Eq. (9)]. For the asphaltene2-based system, direct simulation results reach only half of the result estimated using relaxation time (open diamonds). One possible reason for the difference is the short time (relative to relaxation time) that could be achieved, rendering the Green-Kubo estimate inaccurate. Thus we have more confidence in the relaxation time calculations at different temperatures and in the estimates of low temperature viscosity based on $443.15 \mathrm{~K}$ viscosity simulation results. Bedrov et al. ${ }^{26}$ expressed similar confidence in the estimated viscosity at the lowest temperature they simulated.

The semiquantitive viscosity predictions for naphthalene justify extending this approach to lower temperatures. Because asphaltene has the longest relaxation time, we used its relaxation time ratio to estimate the temperature dependence of the model asphalt systems. Based on the $\tau_{c}$ results in Fig. 10 , we calculated the extent that relaxation time increased as temperature decreased from $443.15 \mathrm{~K}$. Applying Eq. (9), including the temperature factor and neglecting the temperature dependence of the prefactor terms, leads to the asphalt viscosity estimates at 358.15 and $298.15 \mathrm{~K}$ shown in Fig. 14.

The simulation predictions based on Eq. (9) are compared in Fig. 14 with experimental data from the literature for a sample of PG 64-22 asphalt tested by Zhai and Salomon, ${ }^{15}$ for asphalt samples measured by Khong et al. ${ }^{11}$ and for SHRP core asphalts. ${ }^{65}$ The PG 64-22 asphalts always have a higher viscosity than predicted for the asphaltene1and asphaltene2-based systems. Their viscosity-temperature dependences are similar, however. The range of viscosities spanned by the model asphalts at room temperature, based on the estimates using rotational relaxation time, surround the SHRP core asphalt viscosities, considering error bars from Fig. 10. The asphaltene2-based system viscosity is 
somewhat lower, while the asphaltene1-based system has a slightly higher viscosity than SHRP core asphalts. The sample asphalts measured by Khong et al. have viscosities between those calculated for the asphaltene 1 and asphaltene 2 systems, with similar viscosity temperature dependences. The present estimations have uncertainties, as shown in Fig. 10 for relaxation time, and absolute errors for viscosity, as suggested by the results in Fig. 7 for naphthalene. The important result is that the viscosity-temperature trend estimated using rotational relaxation time is comparable to the temperature trend measured experimentally, with viscosity at $298 \mathrm{~K}$ reaching a reasonable value.

Self-consistency among the diffusion coefficient, relaxation time, and viscosity can be compared with the findings of others. Based on the Rouse model in the temperature range we studied, if we neglect the temperature dependence of the density and radius of gyration, the product of viscosity and diffusion coefficient should almost be a constant at a specific temperature. Higher viscosity will correspond to lower diffusion coefficient, which is reasonable. Our simulation results tested that relationship. We observed that the temperature dependence (i.e., activation energy) of the diffusion coefficient and rotational relaxation time are different. Comparing the different compounds, asphaltene molecules have the biggest difference in temperature dependence between the diffusion coefficient and relaxation time. This contrasts with the consistent dependence found in simulations by Gordon of smaller molecules. ${ }^{27,28}$ This is also counter to expectations of a similar dependence for polymers, such as suggested by the Rouse model. This is consistent, however, with results of Mondello and Grest, ${ }^{21}$ who found a more accurate viscosity estimate based on the rotational relaxation time than on diffusivity for moderate length alkanes. The reason could be that asphaltenes mainly are built from fused aromatic rings, so assumptions that depend on the conformational flexibility of polymers break down. For small molecules, the deviations in changes between relaxation time and diffusion coefficient are much smaller: 1-2 orders of magnitude. $n-\mathrm{C}_{22}$ is not a long enough molecule to reach asymptotic scaling regimes, so these differences in temperature dependence are not surprising.

Based on the Tammann criterion, ${ }^{19}$ a liquid turns into a glasslike state at the temperature where the viscosity reaches $10^{13}$ poise. Based on our viscosity estimates at room temperature, the model asphalt systems have not reached a glass transition temperature yet. This is consistent with our earlier analysis based on density results, ${ }^{7}$ in which we estimated a glass transition over short times around or lower than 298.15 $\mathrm{K}$. Here we find VFT $T_{0}$ values approximately $100 \mathrm{~K}$ lower.

In practice, viscosity that is too high at room temperature can lead to poor asphalt mechanical properties at low temperatures, while too low a viscosity at high temperatures could lead to rutting of the road surface. ${ }^{2}$ Modifying the viscosity temperature dependence is useful for improving road performance of asphalts. Adding polymer into asphalts is one common practical choice; the simulation of polymermodified model asphalts will be described in future publications. ${ }^{33,64}$

\section{CONCLUSIONS}

In order to analyze viscosity and local dynamics in asphalts, we applied a molecular dynamics simulation at constant temperature and volume to two three-component model asphalt systems, based on two different asphaltenes. Local dynamics (decay in rotational correlation function and single-molecule diffusion coefficient) were studied at four different temperatures $(443.15,400,358.15$, and $298.15 \mathrm{~K})$. The Green-Kubo and Einstein methods were applied to calculate viscosity at high temperatures (443.15 and $400 \mathrm{~K})$. The Debye-Stokes-Einstein equation and Rouse model were used to inter-relate these results.

Comparing different Legendre polynomials, the third order polynomial of the rotational correlation function decays to zero the fastest. In the model asphalt systems, the Debye rule is satisfied over times less than $1 \mathrm{~ns}$, particularly over times less than $100 \mathrm{ps}$, indicating that different order polynomials of correlation functions will lead to comparable results. Thus using the third order polynomial of the rotational correlation function to calculate the relaxation time is feasible.

First, naphthalene was studied as a simple example of aromatic compounds. Its rotational relaxation time increased smoothly with decreasing temperature. Viscosities calculated using the Green-Kubo and Einstein methods led to results very close to experimental values within simulation uncertainties. The temperature dependence of rotational relaxation time (VFT and Debye-Stokes-Einstein equations) led to comparable viscosity estimates.

The correlation function results were described well by a modified KWW function for naphthalene and model asphalt systems. Analyzing the mKWW function parameters showed that as temperature decreases, $\alpha$ and $\beta$ decrease, while $\tau_{0}$, $\tau_{\mathrm{KWW}}$, and total relaxation time increase. Their relaxation time temperature dependence can be described by the VFT equation. Different molecules have different sensitivity of local dynamics to medium viscosity. 1,7-dimethylnaphthalene in a pure system has the smallest relaxation time; in model asphalt systems, its relaxation time increased. Asphaltene molecules have the strongest relaxation time temperature dependence among the three components, while dimethylnaphthalene molecules have the weakest $\tau_{c}$ temperature dependence; $n$ - $\mathrm{C}_{22}$ molecules are in the middle.

Using mean-squared displacement of the center of mass, we calculated diffusion coefficients for naphthalene and for each component in the model asphalt systems. Asphaltene molecules diffused the slowest, dimethylnaphthalene molecules diffused the fastest, while $n-\mathrm{C}_{22}$ molecules were in between. These differences in diffusion coefficients are related to molecule shape and size. The relaxation time and diffusion coefficient temperature dependences correspond to activation energy. The difference in temperature dependence between diffusion coefficient and relaxation time was largest for asphaltene molecules and was smallest for dimethylnaphthalene molecules. These differences contrast some earlier findings for pure systems of smaller molecules and expectations of theories such as the Rouse model; other earlier works found similar differences. For naphthalene molecules, 
the diffusion coefficient and relaxation time temperature dependences were very similar, with diffusion coefficient activation energy a little higher, and its diffusion coefficient was always higher than any components in the model asphalt systems.

The Green-Kubo and Einstein methods were used to calculate viscosity of the model asphalt systems. Both systems showed similar viscosities at 443.15 and $400 \mathrm{~K}$. The 443.15 $\mathrm{K}$ viscosity and the relaxation time results at different temperatures were used with the Debye-Stokes-Einstein equation to estimate the viscosity at lower temperatures (400, 358.15 , and $298.15 \mathrm{~K}$ ). All were compared with literature data for SHRP core asphalts, for sample asphalts measured by Khong et al., and for a PG 64-22 asphalt. We found that our model asphalt systems have a predicted viscosity at $298.15 \mathrm{~K}$ of the same order of magnitude as SHRP core asphalts and a similar viscosity temperature dependence as sample asphalts studied by Khong et al. A viscosity increase of $10^{10.1}$ for asphaltene1- and $10^{6.9}$ for asphaltene2-based systems between 443.15 and $298.15 \mathrm{~K}$ was estimated, within simulation uncertainties. The overall approach employed here shows promise for assessing the underlying effects that chemical change and polymer modification invoke on asphalt viscosity.

\section{ACKNOWLEDGMENT}

This work was supported through grants from the Rhode Island Department of Transportation (Research and Technology Division) and the University of Rhode Island Transportation Center. We thank Manolis Doxastakis for conversations and ideas about nonlinear regression.

${ }^{1}$ I. A. Wiehe and K. S. Liang, Fluid Phase Equil. 117, 201 (1996).

${ }^{2}$ F. L. Roberts, P. S. Kandhal, E. R. Brown, D.-Y. Lee, and T. W. Kennedy, Hot Mix Asphalt Materials, Mixture Design, and Construction, 2nd ed. (National Asphalt Pavement Association, Lanham, MD, 1996).

${ }^{3}$ J. C. Petersen, R. E. Robertson, J. F. Branthaver, P. M. Harnsberger, J. J. Duvall, S. S. Kim, D. A. Anderson, D. W. Christiansen, and H. U. Bahia, Tech. Rep. SHRP-A-367, Strategic Highway Research Program, 1994, available on-line from http://gulliver.trb.org/publications/shrp/SHRP-A367.pdf

${ }^{4}$ T. W. Kennedy, G. A. Huber, E. T. Harrigan, R. J. Cominsky, C. S. Hughes, H. Von Quintus, and J. S. Moulthrop, Tech. Rep. SHRP-A-410, Strategic Highway Research Program, 1994, available on-line from http://gulliver.trb.org/publications/shrp/SHRP-A-410.pdf

${ }^{5}$ J. A. D'Angelo and F. Fee, J. Assoc. Asph. Paving Technol. 69, 697 (2000).

${ }^{6}$ M. O. Marasteanu, T. Clyne, J. McGraw, X. Li, and R. Velasquez, Transp. Res. Rec. 1901, 52 (2005).

${ }^{7}$ L. Zhang and M. L. Greenfield, Energy Fuels 21, 1712 (2007).

${ }^{8}$ L. Zhang and M. L. Greenfield, Energy Fuels 21, 1102 (2007).

${ }^{9}$ M. Herrin and G. E. Jones, J. Assoc. Asph. Paving Technol. 32, 82 (1963).

${ }^{10}$ S. Glasstone, K. J. Laidler, and H. Eyring, The Theory of Rate Processes (McGraw-Hill, New York, 1941).

${ }^{11}$ T. D. Khong, S. L. Malhotra, and L.-P. Blanchard, Rheol. Acta 17, 654 (1978).

${ }^{12}$ M. L. Williams, R. F. Landel, and J. D. Ferry, J. Am. Chem. Soc. 77, 3701 (1955)

${ }^{13}$ D. W. Christensen, Jr., H. U. Bahia, and D. A. Anderson, J. Assoc. Asph. Paving Technol. 65, 385 (1996).

${ }^{14}$ M. O. Marasteanu and D. A. Anderson, J. Assoc. Asph. Paving Technol. 65, 408 (1996).

${ }^{15}$ H. Zhai and D. Salomon, Transp. Res. Rec. 1901, 44 (2005).

${ }^{16}$ H. Vogel, Phys. Z. 22, 645 (1921).
${ }^{17}$ G. S. Fulcher, J. Am. Ceram. Soc. 8, 339 (1925).

${ }^{18}$ G. Tammann and W. Hesse, Z. Anorg. Allg. Chem. 156, 245 (1926).

${ }^{19}$ C. A. Angell, K. L. Ngai, G. B. McKenna, P. F. McMillan, and S. W. Martin, J. Appl. Phys. 88, 3113 (2000).

${ }^{20}$ M. P. Allen and D. J. Tildesley, Computer Simulation of Liquids (Oxford University Press, New York, 1987).

${ }^{21}$ M. Mondello and G. S. Grest, J. Chem. Phys. 106, 9327 (1997).

${ }^{22}$ M. Mondello, G. S. Grest, E. B. Webb III, and P. Peczak, J. Chem. Phys. 109, 798 (1998).

${ }^{23}$ S. T. Cui, P. T. Cummings, and H. D. Cochran, Mol. Phys. 93, 117 (1998).

${ }^{24}$ P. A. Gordon, Mol. Simul. 29, 479 (2003).

${ }^{25}$ S. T. Cui, S. A. Gupta, P. T. Cummings, and H. D. Cochran, J. Chem. Phys. 105, 1214 (1996).

${ }^{26}$ D. Bedrov, G. D. Smith, and T. D. Sewell, J. Chem. Phys. 112, 7203 (2000).

${ }^{27}$ P. A. Gordon, Ind. Eng. Chem. Res. 42, 7025 (2003).

${ }^{28}$ P. A. Gordon, Ind. Eng. Chem. Res. 44, 5828 (2005).

${ }^{29}$ J. Budzien, C. Raphael, M. D. Ediger, and J. J. de Pablo, J. Chem. Phys. 116, 8209 (2002).

${ }^{30}$ M. L. Greenfield and L. Zhang, Tech. Rep., University of Rhode Island Transportation Center, 2007, to be available on-line via http:// www.uritc.uri.edu.

${ }^{31}$ L. Artok, Y. Su, Y. Hirose, M. Hosokawa, S. Murata, and M. Nomura, Energy Fuels 13, 287 (1999).

${ }^{32}$ H. Groenzin and O. C. Mullins, Energy Fuels 14, 677 (2000).

${ }^{33}$ L. Zhang and M. L. Greenfield, Energy Fuels (submitted).

${ }^{34}$ D. A. Storm, S. J. DeCanio, M. M. DeTar, and V. P. Nero, Fuel 69, 735 (1990).

${ }^{35}$ D. A. Storm, J. C. Edwards, S. J. DeCanio, and E. Y. Sheu, Energy Fuels 8, 561 (1994)

${ }^{36}$ W. L. Jorgensen, D. S. Maxwell, and J. Tirado-Rives, J. Am. Chem. Soc. 118, 11225 (1996).

${ }^{37}$ N. A. McDonald and W. L. Jorgensen, J. Phys. Chem. 102, 8049 (1998).

${ }^{38}$ S. J. Plimpton, 2007, LAMMPS home page, http://lammps.sandia.gov/.

${ }^{39}$ S. J. Plimpton, J. Comput. Phys. 117, 1 (1995).

${ }^{40}$ J.-P. Ryckaert, I. R. McDonald, and M. L. Klein, Mol. Phys. 67, 957 (1989).

${ }^{41}$ M. G. Martin, Fluid Phase Equil. 248, 50 (2006).

${ }^{42}$ M. G. Martin, 2001, TOWHEE home page http://towhee.sourceforge.net.

${ }^{43}$ V. N. Kabadi and W. A. Steele, Mol. Simul. 4, 371 (1990).

${ }^{44}$ W. F. van Gunsteren and M. Karplus, Macromolecules 15, 1528 (1982).

${ }^{45}$ J. P. Hansen and I. R. McDonald, Theory of Simple Liquids (Academic, New York, 1986).

${ }^{46}$ J. L. Dote, D. Kivelson, and R. N. Schwartz, J. Phys. Chem. 85, 2169 (1981).

${ }^{47}$ R. E. Wilde, J. Chem. Phys. 71, 3263 (1979).

${ }^{48}$ M. Doi and S. F. Edwards, The Theory of Polymer Dynamics (Oxford University Press, New York, 1986).

${ }^{49}$ H. Groenzin and O. C. Mullins, J. Phys. Chem. A 103, 11237 (1999).

${ }^{50}$ L. Zhang, Ph.D. thesis, University of Rhode Island, 2007.

${ }^{51}$ G. Williams and D. C. Watts, Trans. Faraday Soc. 66, 80 (1970).

${ }^{52}$ G. Williams and D. C. Watts, Trans. Faraday Soc. 67, 1323 (1971).

${ }^{53}$ M. Doxastakis, D. N. Theodorou, G. Fytas, F. Kremer, R. Faller, and N. Hadjichristidis, J. Chem. Phys. 119, 6883 (2003).

${ }^{54}$ P. J. Turner, 2007, Grace home page, http://plasma-gate.weizmann.ac.il/ Grace.

${ }^{55}$ P. J. Daivis and D. J. Evans, J. Chem. Phys. 100, 541 (1994).

${ }^{56}$ D. N. Theodorou, T. D. Boone, L. R. Dodd, and K. F. Mansfield, Makromol. Chem., Theory Simul. 2, 191 (1993).

${ }^{57}$ D. Frenkel and B. Smit, Understanding Molecular Simulation, 2nd ed. (Academic, San Diego, 2002).

${ }^{58}$ B. S. Gurevich and V. M. Bednov, Zh. Fiz. Khim. 46, 2673 (1972).

${ }^{59}$ L. R. Grzyll, C. Ramos, and D. D. Back, J. Chem. Eng. Data 41, 446 (1996).

${ }^{60}$ CRC Handbook of Chemistry and Physics, 65th ed. (CRC Press, Boca Raton, FL, 1984).

${ }^{61}$ J. A. Dean, Lange's Handbook of Chemistry, 13th ed. (McGraw-Hill, New York, 1985).

${ }^{62}$ E. von Meerwall and S. Beckman, J. Chem. Phys. 108, 4299 (1998). 
${ }^{63}$ M. L. Greenfield and D. N. Theodorou, Macromolecules 26, 5461 (1993).

${ }^{64}$ L. Zhang and M. L. Greenfield, J. Chem. Phys. (unpublished).

${ }^{65}$ R. E. Robertson, J. F. Branthaver, P. M. Harnsberger, J. C. Petersen, S. M.
Dorrence, J. F. McKay, T. F. Turner, A. T. Pauli, S.-C. Huang, J.-D. Huh, J. E. Tauer, K. P. Thomas, D. A. Netzel, F. P. Miknis, T. Williams, J. J. Duvall, F. A. Barbour, and C. Wright, Tech. Rep. FHWA-RD-99-212, Federal Highway Administration, 2001. 